\title{
Theoretical Models of the Planetary Nebula Populations in Galaxies
}

\author{
M. G. Richer ${ }^{1}$, M. L. McCall ${ }^{2}$ and N. Arimoto ${ }^{3}$ \\ ${ }^{1}$ Observatoire de Meudon; ${ }^{2}$ York University; ${ }^{3}$ University of Tokyo
}

We consider the possibility of using bright planetary nebulae to study the chemical evolution of their parent galaxies. In particular, we would like to use planetary nebulae as abundance probes in galaxies where star formation ended long ago, such as ellipticals or the bulges of spirals, and in which no other direct probes exist. However, if we are to use planetary nebulae to study the chemical evolution of their parent galaxies, we must relate the oxygen abundance in bright planetary nebulae to the oxygen abundance in the interstellar medium when star formation stopped. Obviously, only theoretical modelling can provide this information once star formation has stopped.

We model the chemical evolution of the parent galaxy using the code described by Arimoto, Yoshii, \& Takahara (1992). We then use the star formation history provided by this code to determine which stellar populations are producing planetary nebulae. These planetary nebulae are then evolved through the HR diagram to determine the distribution of their [O III] $\lambda 5007$ luminosities. Once the [O III] $\lambda 5007$ luminosity distribution is known for the planetary nebulae from each stellar population, we calculate the mean oxygen abundance for samples of planetary nebulae in various luminosity bins. Initially, we adopt a rather simple planetary nebula evolution scheme: the central stars follow hydrogen-burning evolutionary tracks, and the envelopes have a uniform density and a constant expansion velocity.

These models reproduce a constant planetary nebula luminosity function (PNLF) peak luminosity, the PNLF shape in all galaxies, as well as the mean nebular densities and mean oxygen abundances observed in the Magellanic Clouds. However, to achieve this, we found it necessary to impose a mass-dependent nebular covering factor and to couple the evolution of the nebular shell and the central star. The first of these modifications was required to prevent the planetary nebulae from young progenitors in star-forming galaxies from having luminosities far in excess of the maximum luminosity observed. The second modification was required to match the PNLF shape in ellipticals and the bulges of spirals, and to match the nebular densities and masses observed in the Magellanic Clouds.

These planetary nebula population models predict that a gap develops between the abundances observed in bright planetary nebulae and those in the interstellar medium when star formation stopped. This abundance gap depends primarily upon the oxygen abundance achieved in the interstellar medium when star formation stopped, though it also has some sensitivity to the history of star formation. For the Milky Way, the predicted abundance gap, 0.14 dex, is identical to that observed. Richer, McCall, \& Arimoto (1996) use these models to investigate whether spheroidal galaxies are evolutionary relatives of dwarf irregulars.

\section{REFERENCES}

Arimoto, N., Yoshii, Y., \& Takahara, F. 1992, A\&A, 253, 34

Richer, M. G., McCall, M. L., \& Arimoto, N. 1996, A\&AS, in press 